Vol. 56, 7. 1-2 - 2003

s. $61-76$

\title{
The influence of heterostyly, pollination method and hormonization on eggplant's (Solanum melongena $\mathrm{L}$.) flowering and fruiting
}

\author{
GRAŻYNA KOWALSKA \\ Department of Vegetable and Medicinal Plants Agricultural University, \\ Leszczyńskiego St 58. 20-068 Lublin. Poland
}

(Received: 17.01.2003)

\section{Summary}

The experiment was carried out in a three unheated plastic tunnels in 19982000. The aim of this study was to estimate the effects of flower's heterostyly and two methods of flower pollination (self-pollination and using bumble-bee) as well as flower hormonization on the flowering and fruiting of three varieties of aubergine 'Black Beauty', 'Solara $F_{1}$ and 'Epic $F_{1}$ '. The analysis of results showed that the eggplants formed more flowers in object with self-pollination and flower hormonization than those pollinated by bumble-bee. Regardless of the pollination way and flower hormonization, eggplants formed the highest number of flowers with long pistil and much less - with medium and short pistil. It was shown that the tendency to formation the flowers with particular type of pistil is the variety trait of eggplants. The highest number of flowers with long pistil was observed in varieties 'Solara $F_{1}$ ' and 'Epic $\mathrm{F}_{1}$ ', and those with medium pistil - in 'Black Beauty' variety.

Heterostyly phenomenon occurring in eggplant's flowers affected the plant's fruiting. The most fruits were set from flowers with long pistils than from those with medium and short ones. Fruits formed from long pistil flowers were characterized with significantly greater mean weight and size, than those formed from medium and short pistil ones.

No significant influence of pollination method on eggplant fruit quality was found in three years of study. Fruits achieved due to three pollination methods were characterized with similar mean weight and diameter. Fruits with significantly larger mean length were achieved from flowers pollinated by bumble-bees than from self pollinated ones.

Key words: eggplant, methods of pollination, bumble-bees, growth regulator, self-pollination, heterostyly, flowering. fruiting 


\section{INTRODUCTION}

The eggplant (Solanum melongena L.) also known as an aubergine, is a vegetable, whose an edible part, juicy berry, has a variable shape. In our climatic conditions a satisfactory yield of that vegetable is obtained only if it is grown under covers. Due to inhibition of pollen germination, insufficient fruit setting and much reduced fruit development occur under conditions of too high or too low temperature (Nothmann and Koller, 1975). Phenomenon of short pistil formation, that due to low absorption of stigmates are not pollinated and plants do not set fruit, is an additional factor reducing yielding potential of many eggplant varieties (Pas a m and Bolmatis , 1997). Taking into account the variability of microclimate conditions under cover and eggplant flower's morphology, it was found out that better pollination and fruit setting can be achieved introducing growth regulators into eggplant cultivation. Insects, among which different bumble-bee varieties appeared to be excellent pollinators of vegetables cultivated under cover, are also considered (Nothman $\mathrm{n}$ et al., 1983; Shipp et al., 1994; Abak et al., 1995; Dobromilska, 1997).

\section{MATERIAL AND METHODS}

In the years 1998-2000 the experiment was conducted in a cold plastic tunnel on the Experimental Farm of Agricultural University in Lublin. Potted seedlings were maintained in glasshouse culture, according to standard recommendations for that species. They were transplanted into a tunnel at the beginning of June. Spacing between plants was $50 \times 50 \mathrm{~cm}$. Eggplant plants were grown in plastic cylinders, each of 8 litres volume, in a peat substrate. Fertiliser application (pretransplant and sidedressing) was based on prior soil test.

Plants of three eggplant (Solanum melongena L.) varieties - 'Black Beauty', 'Solara $F_{1}$ ' and 'Epic $F_{1}$ ' that were cultivated in three foil tunnels (120 plants each) were used for study. In the first tunnel, plants whose fruits were set due to flower pollination by insects (Bombus terrestris) were placed. Net curtains that made possible for insects to exit the tunnel were mounted at the entrance. Beehive with bumblebee colony was introduced twice (with two-week interval) in 1998 and 1999. In 2000 eggplant plants did not grow abundantly and one bumble-bee family was sufficient to pollinate all flowers on plants. The smallest "mini" beehive (Biopartner) assigned for pollination $300 \mathrm{~m}^{2}$ area was used in the experiment. Bumble-bees were introduced onto the cultivation just after first flowers appearance: $1998-25.06$. and 5.08., 1999 - 8.07. and 5.08., $2000-24.06$. Control of flower's pollination by means of pistil's colour observation was made several times a week. Flowers efficiently pollinated had brown-coloured pistils. In the second tunnel, plants whose flowers were subjected to hormonization, were placed. Eggplant's flowers were sprayed with Betokson Super preparation containing 2.5\% $\beta$-naphthoxyacetic acid (NOA). Flower hormonization was begun on $30^{\text {th }}$ June $1998,6^{\text {th }}$ July 1999 and $24^{\text {th }}$ June 2000 repeating the operations twice a week in the morning ( 9 a.m. till 11 a.m.). Control plants were cultivated in the third tunnel. Fruits on these plants were set only due to self-pollination. 
Taking into account the phenomenon of heterostyly in eggplant. also observations of flower morphology were made and flowers with long, medium and short pistil were classified (Photo 1). In each combination, observations were performed using 10 randomly chosen plants. Every flower was marked at full of flowering, including blooming date, pistil length and number of flower on a plant. Then it was checked whether particular flower set a fruit or not.

During the growing season a pest management and cultural practices were standard, recommended ones. Eggplant fruits were collected at a harvesting maturity stage. when they were 40 days old, in two weeks intervals, from the first decade of August to the first decade of October. Then weight, diameter and length of each fruit on plant were measured.

The experiment was established as two-factor one a completely randomised design. Each combination of the factor at stake was represented by 40 experimental units. The yield results were analysed using the analysis of variance. Significant differences were detected with T-Tukey's multiply confidence intervals at $\alpha=0.05$.

\section{RESULTS}

In 1998-2000, the influence of flower's heterostyly and applied operations on eggplant's flowering was studied. Significantly highest mean number of flowers was recorded on plants whose flowers were self-pollinated (28.2 flowers per plant) as compared to those with hormonized flowers (24.1 flowers per plant) and pollinated by insects - 15.9 flowers per plant (Tables 1-3). No significant effect of applied operations on eggplant's fruiting was observed. Mean number of set fruits was 4.6-5.1 flowers per plant (Tables 1-3).

The share of set fruits in the number of flowers produced amounted from $18.1 \%$ in control up to $29.6 \%$ in an object with plants pollinated by insects (Tables 1-3). Taking into account all tested ways of pollination, flowers with long pistil were 42.6$50.9 \%$ of total number of flowers produced on a plant. 20.1-35.1\% flowers with medium pistil and 22.3-28.9\% with short pistil were recorded (Fig. 1). During three study years, the largest number of fruits was set on eggplants with long pistil (64.7$74.5 \%$ ). less with medium pistil (21.3-29.4\%), and 4.3-5.9\% with short pistil (Fig. 2). In each studied combination, varieties 'Solara $\mathrm{F}_{1}$ ' and Epic $\mathrm{F}_{1}$ ' produced the highest number of flowers with long pistil (13.3 and 12.6 flowers per plant) and variety 'Black Beauty ${ }^{\prime}$ - flowers with medium pistil - 12.5 flowers per plant (Tables 1-3).

In 1998-2000, the highest number of flowers varieties 'Solara $F_{1}$ ' and 'Epic $F_{1}$ ' set the most fruits from flowers with long pistil (4.3 and 4.0 fruits per plant), and for variety 'Black Beauty' - from flowers with medium pistil - 2.0 fruits per plant (Tables 1-3). Studied varieties differed referring to total number of flowers and fruits produced. On average, the largest number of flowers was recorded in 'Black Beauty' variety (16.9-33.5 flowers per plant) as compared to varieties: 'Solara $F_{1}$ ' (15.9-24.3 flowers per plant) and 'Epic $F_{1}$ ' (14.9-26.9 flowers per plant). Higher number of fruits per plant was achieved from varieties: 'Epic $F_{1}$ ' and 'Solara $F_{1}$ ' (4.7-6.1 fruits per plant) than from variety 'Black Beauty' (4.0-4.2 fruits per plant) (Tables 1-3). 


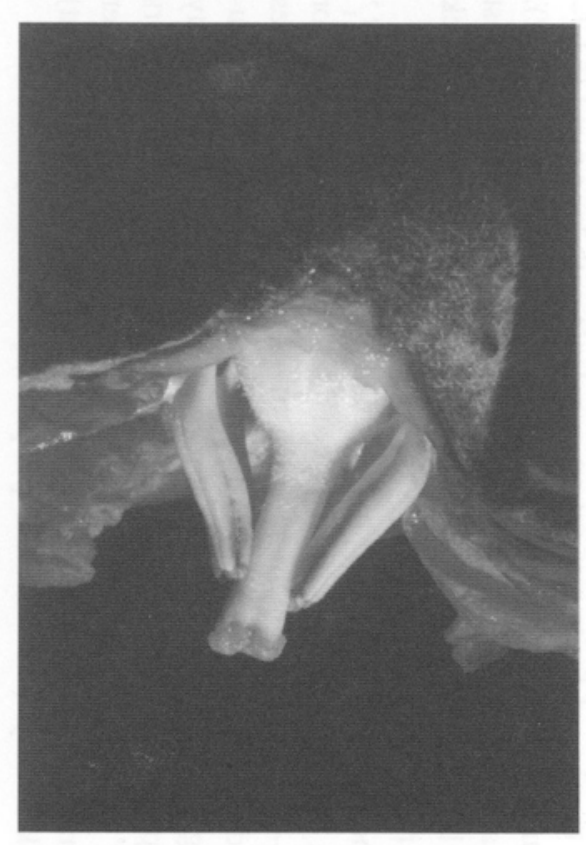

Flower with long pistil

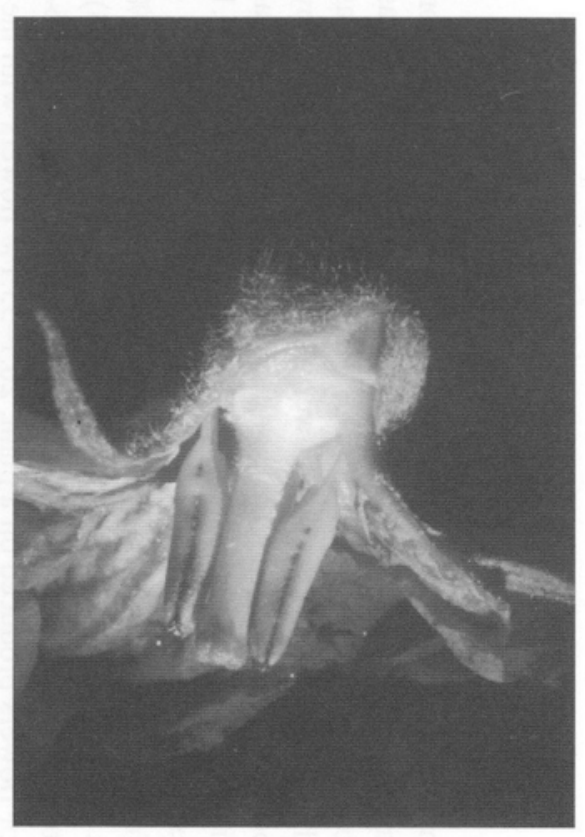

Flower with medium pistil

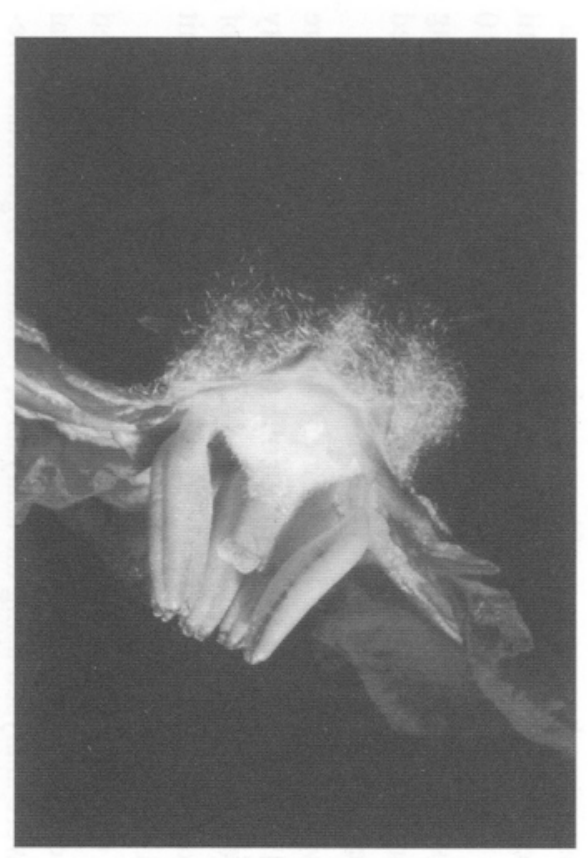

Flower with short pistil

Photo 1. Heterostyly phenomenon occurring in eggplant,s flowers (photo: Author) 
In combinations with self-pollination and hormonization, plants of three eggplant varieties produced more flowers in 1998 (37.5 and 30.3 flowers per plant) than in 1999 (25.4 and 21.1 flowers per plant) and 2000 (21.8 and 21.2 flowers per plant). In object where flowers were pollinated by bumble-bee, plants cultivated in 1999 were characterized with the highest number of flowers produced ( 23.2 flowers per plant) as compared to 1998 (13.8 flowers per plant) and 2000 (10.3 flowers per plant). Referring to pollination ways, the highest number of set fruits was achieved in 1999 (5.16.1 fruits per plant), then in 1998 (4.4-5.0 fruits per plant) and 2000 (3.9-4.2 fruits per plant) (Tables 1-3).

Phenomenon of flower heterostyly influence on mean eggplant fruit weight was found in 1998-2000. Fruits with significantly higher mean weight (341.2 g) were obtained from plants whose flowers had long, than medium pistil (241.4 g). Fruits with significantly lowest mean weight (153.3 g) were achieved from plants with short pistil (Table 4). Heterostyly occurring on eggplant flowers had significant influence on parameters of harvested fruits. Fruits formed from long pistil flowers were characterized with significantly greater length $(15.0 \mathrm{~cm})$ and diameter $(8.1 \mathrm{~cm})$ than those from medium pistil ones (length $-13.1 \mathrm{~cm}$ and diameter $-7.2 \mathrm{~cm}$ ). Significantly smallest fruits (length $-11.0 \mathrm{~cm}$ and diameter $-5.9 \mathrm{~cm}$ ) were obtained from short pistil flowers (Tables 5, 6).

No significant influence of pollination method on eggplant fruits quality was found in three years of study. Fruits achieved due to three pollination methods were characterized with similar mean weight $(256.3-239.9 \mathrm{~g})$ and diameter $(7.1 \mathrm{~cm})(\mathrm{Ta}-$ bles 4,6$)$. Fruits with significantly larger mean length $(13.5 \mathrm{~cm})$ were achieved from flowers pollination by bumble - bees than from self - pollination ones $(12.5 \mathrm{~cm})$ (Table 5).

Significant differences regarding to mean weight and fruit size in commercial yield were recorded. In all study years, fruits with significantly higher weight were achieved at 'Black Beauty' (288.9 g) than at 'Solara F', (226.9 g) and 'Epic F ' (220.1 g) (Table 4). Fruits with significantly larger mean length were achieved from varieties: 'Solara F' (14.4 cm) and 'Epic F'' $(13.4 \mathrm{~cm})$ as compared to 'Black Beauty' $(11.3 \mathrm{~cm})$. Fruits with significantly larger mean fruits diameter were collected from 'Black Beauty' $(8.6 \mathrm{~cm})$ than from 'Epic F' $(6.4 \mathrm{~cm})$ and 'Solara $F_{1}$ ' $(6.2 \mathrm{~cm})$ varieties (Tables 5, 6).

Significant differences referring to mean weight and fruit size among study years were also proved. Fruits with significantly highest mean weight were achieved in 1999 (282.8 g), and with significantly lowest one - in 1998 (215.9 g) and in 2000 $(237.2 \mathrm{~g})$ (Table 4). The biggest fruits (length $13.9 \mathrm{~cm}$, diameter $7.4 \mathrm{~cm}$ ) were harvested in 1999. Fruits achieved in 1998 and 2000 were characterised with lesser size (length -11.7 and $13.5 \mathrm{~cm}$, diameter -7.0 and $6.9 \mathrm{~cm}$, respectively) (Tables 5,6 ). 
Table 1

The influence of heterostyly on eggplant's flowering and fruiting in object with self - pollination

\begin{tabular}{|c|c|c|c|c|c|c|c|c|c|c|c|c|c|}
\hline \multicolumn{14}{|c|}{ Self - pollination } \\
\hline \multirow[t]{2}{*}{ Cultivar } & \multirow[t]{2}{*}{ Pistil length } & \multicolumn{4}{|c|}{ The number of flowers (no/plant) } & \multicolumn{4}{|c|}{ The number of fruits (no/plant) } & \multicolumn{4}{|c|}{$\begin{array}{c}\text { The share of number fruits in the } \\
\text { number of flowers }(\%)\end{array}$} \\
\hline & & 1998 & 1999 & 2000 & $1998-2000$ & 1998 & 1999 & 2000 & $1998-2000$ & 1998 & 1999 & 2000 & $1998-2000$ \\
\hline \multirow{4}{*}{$\begin{array}{l}\text { 'Black } \\
\text { Beauty' }\end{array}$} & Long pistil & 1.3 & 5.6 & 9.8 & 5.6 & 0.2 & 1.5 & 2.2 & 1.3 & 15.4 & 26.8 & 22.4 & 23.2 \\
\hline & Medium pistil & 38.0 & 14.4 & 5.8 & 19.4 & 3.7 & 3.1 & 0.9 & 2.6 & 9.7 & 21.5 & 15.5 & 13.4 \\
\hline & Short pistil & 2.7 & 11.6 & 11.2 & 8.5 & 0.1 & 0.2 & 0.1 & 0.1 & 0.0 & 1.7 & 0.9 & 1.2 \\
\hline & Sum & 42.0 & 31.6 & 26.8 & 33.5 & 3.9 & 4.8 & 3.2 & 4.0 & 9.3 & 15.2 & 11.9 & 11.9 \\
\hline \multirow{4}{*}{ 'Solara $F_{1}$ ' } & Long pistil & 19.8 & 11.3 & 14.1 & 15.1 & 4.2 & 4.7 & 3.8 & 4.2 & 21.2 & 41.6 & 27.0 & 27.8 \\
\hline & Medium pistil & 5.8 & 3.9 & 0.9 & 3.5 & 0.1 & 0.8 & 0.2 & 0.4 & 1.7 & 20.5 & 22.2 & 11.4 \\
\hline & Short pistil & 5.3 & 6.1 & 5.6 & 5.7 & 0.2 & 0.9 & 0.5 & 0.5 & 3.8 & 14.8 & 8.9 & 8.8 \\
\hline & Sum & 30.9 & 21.3 & 20.6 & 24.3 & 4.5 & 6.4 & 4.5 & 5.1 & 14.6 & 30.0 & 21.8 & 21.0 \\
\hline \multirow{4}{*}{ 'Epic $F_{1}$ ' } & Long pistil & 19.3 & 16 & 10.4 & 15.4 & 3.8 & 6.4 & 2.9 & 4.4 & 19.7 & 39.0 & 27.9 & 28.6 \\
\hline & Medium pistil & 15.8 & 1.9 & 2.4 & 6.7 & 2.6 & 0.7 & 0.8 & 1.4 & 16.5 & 36.8 & 33.3 & 20.9 \\
\hline & Short pistil & 4.2 & 5.0 & 5.3 & 4.8 & 0.4 & 0.2 & 0.3 & 0.3 & 9.5 & 4.0 & 5.7 & 6.3 \\
\hline & Sum & 39.3 & 23.3 & 18.1 & 26.9 & 6.8 & 7.3 & 4.0 & 6.1 & 17.3 & 31.3 & 22.1 & 22.7 \\
\hline \multirow{3}{*}{$\bar{x}$} & Long pistil & 13.5 & 11.1 & 11.4 & 12.0 & 2.7 & 4.2 & 3.0 & 3.3 & 20.0 & 37.8 & 26.3 & 27.5 \\
\hline & Medium pistil & 19.9 & 6.7 & 3.0 & 9.9 & 2.1 & 1.5 & 0.6 & 1.5 & 10.6 & 22.4 & 20.0 & 15.2 \\
\hline & Short pistil & 4.1 & 7.6 & 7.4 & 6.3 & 0.2 & 0.4 & 0.3 & 0.3 & 4.9 & 5.3 & 4.1 & 4.8 \\
\hline
\end{tabular}

$\operatorname{LSD}_{005}$

$\begin{array}{lllllll}\text { Cultivar (a) } & 3.65 & 2.26 & 2.46 & 0.85 & 0.80 & \text { ns. }\end{array}$

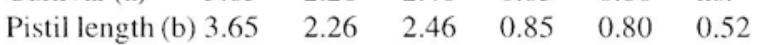

Interaction (ab) $8.45 \quad 5.24 \quad 5.70 \quad 1.97 \quad 1.86 \quad 1.21$ 
Table 2

The influence of heterostyly on eggplant's flowering and fruiting in object with bumble - bees

\begin{tabular}{|c|c|c|c|c|c|c|c|c|c|c|c|c|c|}
\hline \multicolumn{14}{|c|}{ Pollination by bumble - bees } \\
\hline \multirow[t]{2}{*}{ Cultivar } & \multirow[t]{2}{*}{ Pistil length } & \multicolumn{4}{|c|}{ The number of flowers (no/plant) } & \multicolumn{4}{|c|}{ The number of fruits (no/plant) } & \multicolumn{4}{|c|}{$\begin{array}{l}\text { The share of number fruits } \\
\text { in the number of flowers }(\%)\end{array}$} \\
\hline & & 1998 & 1999 & 2000 & $1998-2000$ & 1998 & 1999 & 2000 & $1998-2000$ & 1998 & 1999 & 2000 & $1998-2000$ \\
\hline \multirow{4}{*}{$\begin{array}{l}\text { 'Black } \\
\text { Beauty' }\end{array}$} & Long pistil & 3.8 & 5.1 & 4.2 & 4.4 & 2.4 & 1.4 & 2.4 & 2.1 & 63.2 & 27.5 & 57.1 & 47.7 \\
\hline & Medium pistil & 10.1 & 8.1 & 1.4 & 6.5 & 1.8 & 3.4 & 0.6 & 1.9 & 17.8 & 42.0 & 42.9 & 29.2 \\
\hline & Short pistil & 1.5 & 14.1 & 2.3 & 6.0 & 0.1 & 0.3 & 0.2 & 0.2 & 0.0 & 2.1 & 8.7 & 3.3 \\
\hline & Sum & 15.4 & 27.3 & 7.9 & 16.9 & 4.2 & 5.1 & 3.2 & 4.2 & 27.3 & 18.7 & 40.5 & 24.9 \\
\hline \multirow{4}{*}{ 'Solara $F_{1}$ ' } & Long pistil & 9.6 & 13.2 & 10.0 & 11.3 & 3.6 & 4.6 & 4.8 & 4.7 & 37.5 & 34.8 & 48.0 & 41.6 \\
\hline & Medium pistil & 1.0 & 1.6 & 0.5 & 1.0 & 0.1 & 0.6 & 0.1 & 0.2 & 10.0 & 37.5 & 0.0 & 20.0 \\
\hline & Short pistil & 1.1 & 8.3 & 1.4 & 3.6 & 0.1 & 0.1 & 0.1 & 0.1 & 0.0 & 1.2 & 0.0 & 0.0 \\
\hline & Sum & 11.7 & 23.1 & 11.9 & 15.9 & 3.7 & 5.3 & 4.8 & 4.9 & 31.6 & 22.9 & 40.3 & 30.8 \\
\hline \multirow{4}{*}{ 'Epic Fi' } & Long pistil & 10.6 & 8.7 & 6.9 & 8.7 & 3.8 & 3.7 & 3.8 & 3.8 & 35.8 & 42.5 & 55.1 & 43.7 \\
\hline & Medium pistil & 3.1 & 2.0 & 1.1 & 2.1 & 1.5 & 0.6 & 0.3 & 0.8 & 48.4 & 30.0 & 27.3 & 38.1 \\
\hline & Short pistil & 0.8 & 8.5 & 3.1 & 4.1 & 0.1 & 1.2 & 0.1 & 0.4 & 0.0 & 14.1 & 0.0 & 9.8 \\
\hline & \begin{tabular}{|l} 
Sum \\
\end{tabular} & 14.5 & 19.2 & 11.1 & 14.9 & 5.3 & 5.5 & 4.1 & 5.0 & 36.6 & 28.6 & 36.9 & 33.6 \\
\hline \multirow{4}{*}{$\bar{x}$} & Long pistil & 8.0 & 9.0 & 7.0 & 8.1 & 3.3 & 3.2 & 3.7 & 3.5 & 41.3 & 35.6 & 52.9 & 43.2 \\
\hline & Medium pistil & 4.7 & 3.9 & 1.0 & 3.2 & 1.1 & 1.5 & 0.3 & 1.0 & 23.4 & 38.5 & 30.0 & 31.3 \\
\hline & Short pistil & 1.1 & 10.3 & 2.3 & 4.6 & 0.1 & 0.5 & 0.1 & 0.2 & 0.0 & 4.9 & 4.3 & 4.3 \\
\hline & Sum & 13.8 & 23.2 & 10.3 & 15.9 & 4.4 & 5.2 & 4.1 & 4.7 & 31.9 & 22.4 & 38.8 & 29.6 \\
\hline
\end{tabular}

$\operatorname{LSD}_{0,05}$

Cultivar (a)

Pistil length (b)

ns. $\quad 1.19$

ns.

ns.

ns.

Interaction (ab)

5.24

2.92

1.19

0.83

0.80

0.53

erese

$\begin{array}{lll}1.92 & 1.86 & 1.22\end{array}$


The influence of heterostyly on eggplant's flowering and fruiting in object with flower hormonization

\begin{tabular}{|c|c|c|c|c|c|c|c|c|c|c|c|c|c|}
\hline \multicolumn{14}{|c|}{ Hormonization } \\
\hline \multirow[t]{2}{*}{ Cultivar } & \multirow[t]{2}{*}{ Pistil length } & \multicolumn{4}{|c|}{ The number of flowers (no/plant) } & \multicolumn{4}{|c|}{ The number of fruits (no/plant) } & \multicolumn{4}{|c|}{$\begin{array}{c}\text { The share of number fruits in the } \\
\text { number of flowers }(\%)\end{array}$} \\
\hline & & 1998 & 1999 & 2000 & $1998-2000$ & 1998 & 1999 & 2000 & $1998-2000$ & 1998 & 1999 & 2000 & $1998-2000$ \\
\hline \multirow{4}{*}{$\begin{array}{l}\text { 'Black } \\
\text { Beauty' }\end{array}$} & Long pistil & 3.3 & 4.7 & 8.6 & 5.5 & 1.7 & 1.4 & 3.1 & 2.1 & 51.5 & 29.8 & 36.0 & 38.2 \\
\hline & Medium pistil & 21.2 & 9.2 & 4.8 & 11.7 & 1.9 & 2.6 & 0.3 & 1.6 & 9.0 & 28.3 & 6.3 & 13.7 \\
\hline & Short pistil & 1.3 & 13.1 & 10.3 & 8.2 & 0.1 & 0.6 & 0.2 & 0.3 & 7.7 & 4.6 & 1.9 & 3.7 \\
\hline & Sum & 25.8 & 27.0 & 23.7 & 25.4 & 3.7 & 4.6 & 3.6 & 4.0 & 14.3 & 17.0 & 15.2 & 15.7 \\
\hline \multirow{4}{*}{ 'Solara $F_{1}$ ' } & Long pistil & 15.9 & 10.7 & 14.5 & 13.7 & 3.7 & 3.6 & 4.7 & 4.0 & 23.3 & 33.6 & 32.4 & 29.2 \\
\hline & Medium pistil & 4.8 & 3.5 & 1.7 & 3.3 & 0.7 & 1.6 & 0.5 & 0.9 & 14.6 & 45.7 & 29.4 & 27.3 \\
\hline & Short pistil & 4.0 & 6.0 & 5.1 & 5.0 & 0.4 & 0.4 & 0.1 & 0.3 & 10.0 & 6.7 & 2.0 & 6.0 \\
\hline & Sum & 24.7 & 20.2 & 21.3 & 22.0 & 4.8 & 5.6 & 5.3 & 5.2 & 19.4 & 27.7 & 24.9 & 23.6 \\
\hline \multirow{4}{*}{ 'Epic F' } & Long pistil & 19.0 & 18 & 10.4 & 13.7 & 3.7 & 4.7 & 3.1 & 3.8 & 19.5 & 39.8 & 29.8 & 27.7 \\
\hline & Medium pistil & 13.2 & 1.2 & 1.9 & 5.4 & 1.3 & 0.4 & 0.8 & 0.8 & 9.8 & 33.3 & 42.1 & 14.8 \\
\hline & Short pistil & 8.1 & 3.0 & 6.2 & 5.8 & 0.1 & 0.1 & 0.1 & 0.1 & 1.2 & 3.3 & 1.6 & 1.7 \\
\hline & Sum & 40.3 & 16.0 & 18.5 & 24.9 & 5.1 & 5.2 & 4.0 & 4.7 & 12.7 & 32.5 & 21.6 & 18.9 \\
\hline \multirow{4}{*}{$\mathrm{x}$} & Long pistil & 12.7 & 9.1 & 11.2 & 11.0 & 3.0 & 3.2 & 3.6 & 3.3 & 23.6 & 35.2 & 32.1 & 30.0 \\
\hline & Medium pistil & 13.1 & 4.6 & 2.8 & 6.8 & 1.3 & 1.5 & 0.5 & 1.1 & 28.9 & 32.6 & 17.9 & 16.2 \\
\hline & Short pistil & 4.5 & 7.4 & 7.2 & 6.3 & 0.2 & 0.4 & 0.1 & 0.2 & 4.4 & 5.4 & 1.4 & 3.2 \\
\hline & Sum & 30.3 & 21.1 & 21.2 & 24.1 & 4.5 & 5.1 & 4.2 & 4.6 & 14.9 & 24.2 & 19.8 & 19.1 \\
\hline
\end{tabular}

$\mathrm{LSD}_{0.05}$

$\begin{array}{lllllll}\text { Cultivar (a) } & 3.32 & 2.20 & \text { ns. } & \text { ns. } & 0.45 & \text { ns. }\end{array}$

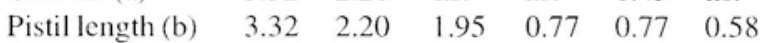

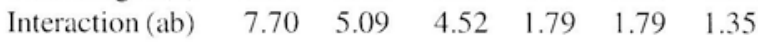




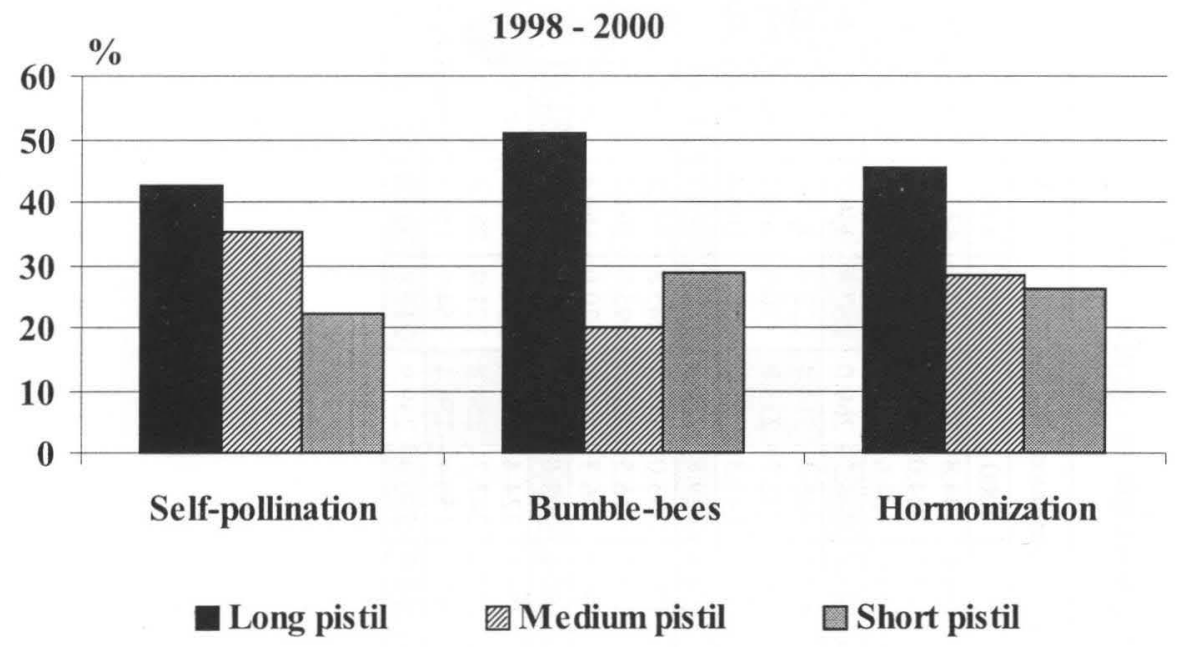

Fig. 1. Share of flowers number with different pistil length in total number of flowers

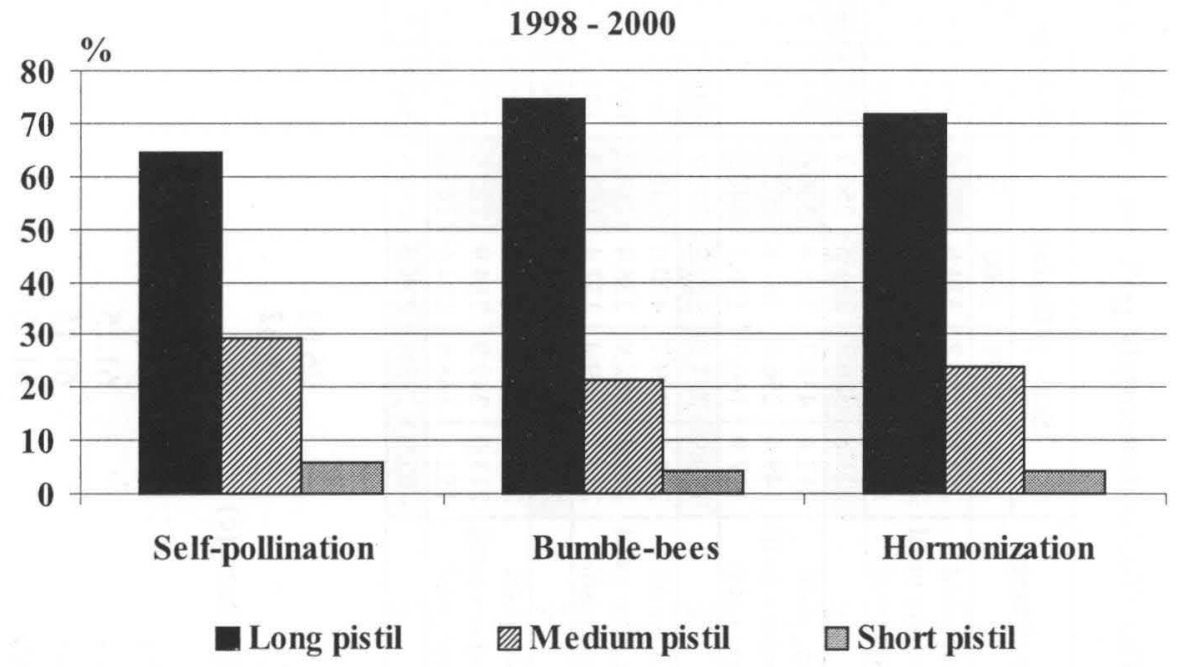

Fig. 2. Share of fruit number set from particular flowers in total number of fruits 
Table 4

The effect of heterostyly, pollination method and hormonization on the mean weight of eggplant fruits (g)

\begin{tabular}{|c|c|c|c|c|c|c|c|c|c|c|c|c|c|c|c|c|c|}
\hline \multirow{2}{*}{ Cultivar } & \multirow{2}{*}{ Pistil length } & \multicolumn{4}{|c|}{ Self-pollination } & \multicolumn{4}{|c|}{ Pollination by bumble - bees } & \multicolumn{4}{|c|}{ Hormonization } & \multicolumn{4}{|c|}{ Mean } \\
\hline & & 1998 & 1999 & 2000 & $\overline{\bar{X}}$ & 1998 & 1999 & 2000 & $\bar{X}$ & 1998 & 1999 & 2000 & $\overline{\bar{X}}$ & 1998 & 1999 & 2000 & $\overline{\mathrm{X}}$ \\
\hline \multirow{4}{*}{$\begin{array}{l}\text { 'Black } \\
\text { Beauty' }\end{array}$} & $\mathrm{L}$ & 253.6 & 539.2 & 324.9 & 372.6 & 554.5 & 476.6 & 460.5 & 497.2 & 225.1 & 400.4 & 344.8 & 323.4 & 344.4 & 472.1 & 376.7 & 397.7 \\
\hline & til & 288.2 & 310.7 & 204.6 & 267.8 & 337.0 & 489.8 & 243.7 & 356.8 & 276.6 & 324.7 & 324.0 & 308.4 & 300.6 & 375.1 & 257.4 & 311.0 \\
\hline & Short pisti & 123.8 & 130.3 & 135.0 & 129.7 & 164.6 & 146.3 & 195.5 & 168.8 & 187.0 & 154.6 & 183.6 & 175.1 & 158.5 & 143.7 & 171.4 & 157.9 \\
\hline & $\bar{x}$ & 221.8 & 326.7 & 221.5 & 256.7 & 352.0 & 370.9 & 299.9 & 340.9 & 229.6 & 293.3 & 284.2 & 269.0 & 267.8 & 330.3 & 268.5 & 288.9 \\
\hline \multirow{4}{*}{ 'Solara Fi' } & Long pis & 212.4 & 431.2 & 348.4 & 330.0 & 251.0 & 439.4 & 284.6 & 325.0 & 203.8 & 394.2 & 345.4 & 314.4 & 222.4 & 421.6 & 326.1 & 323.4 \\
\hline & Medium & 149.4 & 256.0 & 181.8 & 195.7 & 174.8 & 230.0 & 198.0 & 200.9 & 186.0 & 318.6 & 202.7 & 235.8 & 170.1 & 268.2 & 194.2 & 210.8 \\
\hline & Short pistil & 117.9 & 166.3 & 136.3 & 140.2 & 153.3 & 141.2 & 136.3 & 143.6 & 137.5 & 188.6 & 141.3 & 155.8 & 136.2 & 165.4 & 138.0 & 146.5 \\
\hline & $\bar{x}$ & 159.9 & 284.5 & 222.2 & 222.2 & 193.0 & 270.2 & 206.3 & 223.2 & 175.8 & 300.5 & 229.8 & 235.3 & 176.2 & 285.1 & 219.4 & 226.9 \\
\hline \multirow{4}{*}{ 'Epic $F_{1}$ ' } & & 264.5 & 339.7 & 420.0 & 341.5 & 263.8 & 302.7 & 269.7 & 278.7 & 209.1 & 370.0 & 283.0 & 287.4 & 245.8 & 337.5 & 324.2 & 302.5 \\
\hline & lium & 201.4 & 182.9 & 258.4 & 214.2 & 188.8 & 171.9 & 156.0 & 172.2 & 194.9 & 249.5 & 216.5 & 220.3 & 195.0 & 201.4 & 210.3 & 202.2 \\
\hline & Short pistil & 214.7 & 149.3 & 132.4 & 165.5 & 143.5 & 195.0 & 150.8 & 163.1 & 151.9 & 136.9 & 126.3 & 138.4 & 170.0 & 160.4 & 136.5 & 155.7 \\
\hline & $\overline{\bar{X}}$ & 226.9 & 224.0 & 270.3 & 240.4 & 198.7 & 223.2 & 192.2 & 204.7 & 185.3 & 252.1 & 208.6 & 215.3 & 203.6 & 233.1 & 223.7 & 220.1 \\
\hline \multirow{4}{*}{$\bar{x}$} & ng pis & 243.5 & & 364.5 & 348.2 & 356.4 & 406.2 & 338.3 & 367.0 & 212.6 & 388.1 & 324.4 & 308.4 & 270.8 & 410.3 & 342.4 & 341.2 \\
\hline & & 213.0 & 249.9 & 214.9 & 225.9 & 233.5 & 297.2 & 199.2 & 243.3 & 219.2 & 297.6 & 247.7 & 254.8 & 221.9 & 281.6 & 220.6 & 241.4 \\
\hline & Short pistil & 152.1 & 148.6 & 134.6 & 145.1 & 153.8 & 160.8 & 160.8 & 158.5 & 158.8 & 160.1 & 150.4 & 156.4 & 154.9 & 156.5 & 148.6 & 153.3 \\
\hline & $\bar{x}$ & 202.9 & 278.4 & 238.0 & 239.8 & 247.9 & 288.1 & 232.8 & 256.3 & 196.9 & 281.9 & 240.9 & 239.9 & 215.9 & 282.8 & 237.2 & 245.3 \\
\hline
\end{tabular}

$\mathrm{LSD}_{0,05}$

Cultivar (a)

26.62

Pistil length (b)

26.62

Method of pollination (c)

ns.

Years (d)

26.62

Interaction (ab)

61.15

(ac)

61.15

(ad)

61.15

(bd)

61.15 
Table 5

The effect of heterostyly, pollination method and hormonization on the mean length of eggplant fruits (cm)

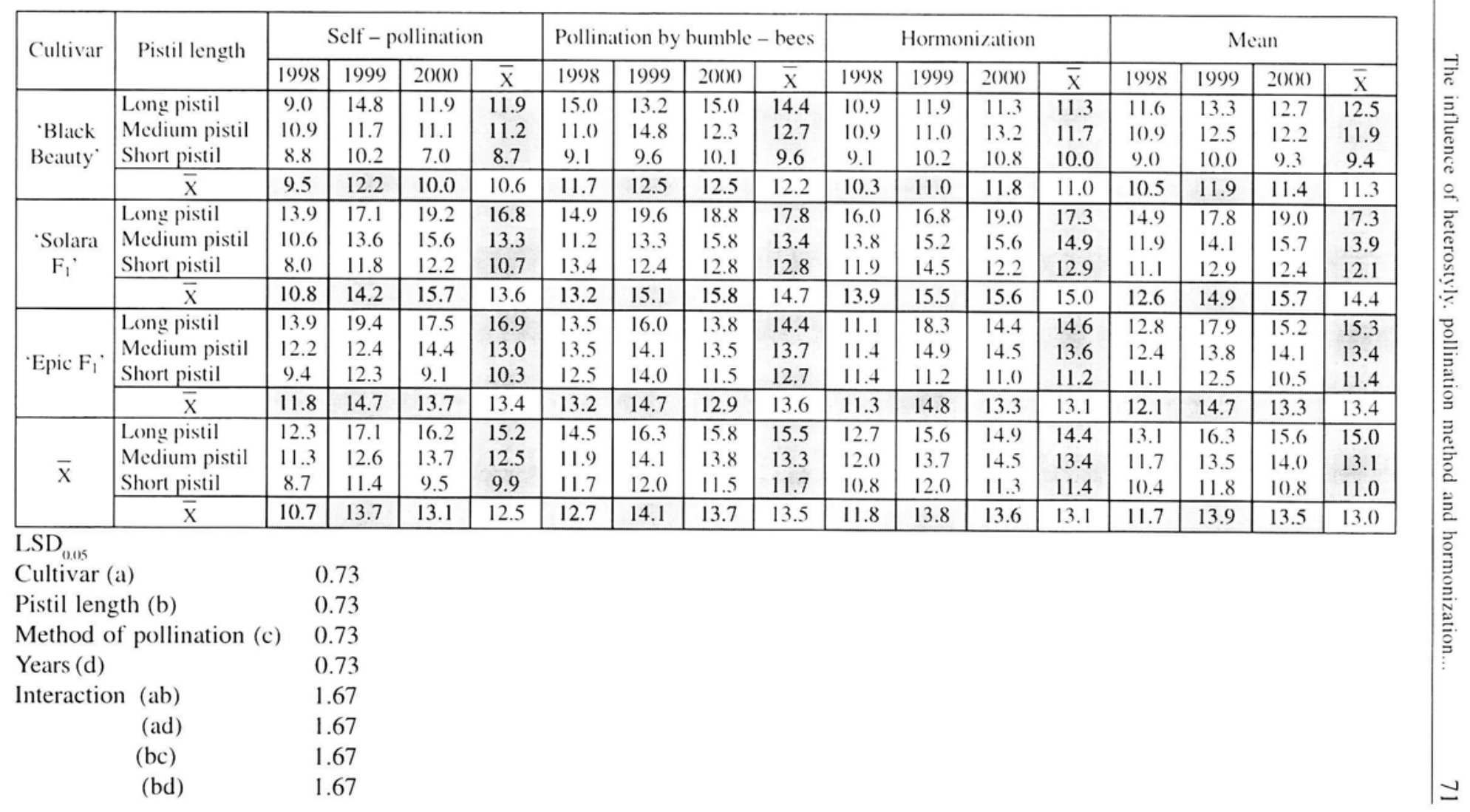


Table 6

The effect of heterostyly, pollination method and hormonization on the mean diameter of eggplant fruits (cm)

\begin{tabular}{|c|c|c|c|c|c|c|c|c|c|c|c|c|c|c|c|c|c|}
\hline \multirow{2}{*}{ Cultivar } & \multirow{2}{*}{ Pistil length } & \multicolumn{4}{|c|}{ Self - pollination } & \multicolumn{4}{|c|}{ Pollination by bumble - bees } & \multicolumn{4}{|c|}{ Hormonization } & \multicolumn{4}{|c|}{ Mean } \\
\hline & & 1998 & 1999 & 2000 & $\bar{x}$ & 1998 & 1999 & 2000 & $\bar{x}$ & 1998 & 1999 & 2000 & $\bar{x}$ & 1998 & 1999 & 2000 & $\bar{x}$ \\
\hline \multirow{4}{*}{$\begin{array}{r}\text { 'Black } \\
\text { Beauty' }\end{array}$} & Long pistil & 10.9 & 10.7 & 9.9 & 10.5 & 12.8 & 10.4 & 10.1 & 11.1 & 8.0 & 10.1 & 11.2 & 9.8 & 10.5 & 10.4 & 10.4 & 10.5 \\
\hline & Medium pistil & 9.1 & 8.9 & 7.9 & 8.6 & 9.6 & 10.5 & 7.9 & 9.3 & 8.5 & 9.2 & 8.6 & 8.8 & 9.1 & 9.5 & 8.1 & 8.9 \\
\hline & Short pistil & 5.4 & 5.9 & 7.1 & 6.1 & 6.1 & 6.5 & 7.4 & 6.7 & 7.0 & 6.1 & 7.5 & 6.9 & 6.2 & 6.2 & 7.3 & 6.6 \\
\hline & $\bar{x}$ & 8.5 & 8.5 & 8.3 & 8.4 & 9.5 & 9.1 & 8.5 & 9.0 & 7.8 & 8.5 & 9.1 & 8.5 & 8.6 & 8.7 & 8.6 & 8.6 \\
\hline \multirow{4}{*}{ 'Solara Fi' } & Long pistil & 6.2 & 9.0 & 6.0 & 7.1 & 6.3 & 7.1 & 6.4 & 6.6 & 5.8 & 7.9 & 6.5 & 6.7 & 6.1 & 8.0 & 6.3 & 6.8 \\
\hline & Medium pistil & 5.4 & 7.6 & 5.5 & 6.2 & 5.9 & 6.9 & 5.6 & 6.1 & 5.6 & 7.9 & 5.8 & 6.4 & 5.6 & 7.5 & 5.6 & 6.2 \\
\hline & Short pistil & 5.5 & 5.8 & 5.5 & 5.6 & 5.3 & 5.5 & 5.3 & 5.3 & 5.2 & 6.5 & 5.5 & 5.8 & 5.3 & 5.9 & 5.4 & 5.6 \\
\hline & $\bar{x}$ & 5.7 & 7.5 & 5.7 & 6.3 & 5.8 & 6.5 & 5.8 & 6.0 & 5.5 & 7.4 & 5.9 & 6.3 & 5.7 & 7.1 & 5.8 & 6.2 \\
\hline \multirow{4}{*}{ 'Epic $F_{1}$ ' } & Long pistil & 6.6 & 7.1 & 7.4 & 7.0 & 7.4 & 7.2 & 6.8 & 7.1 & 8.0 & 7.8 & 6.6 & 7.5 & 7.3 & 7.4 & 6.9 & 7.2 \\
\hline & Medium pistil & 7.3 & 6.5 & 6.8 & 6.8 & 6.0 & 5.6 & 5.8 & 5.8 & 7.5 & 6.5 & 6.1 & 6.7 & 6.9 & 6.2 & 6.2 & 6.4 \\
\hline & Short pistil & 6.0 & 5.6 & 5.8 & 5.8 & 5.5 & 6.1 & 5.5 & 5.7 & 5.9 & 5.2 & 5.0 & 5.4 & 5.8 & 5.7 & 5.4 & 5.6 \\
\hline & $\bar{x}$ & 6.6 & 6.4 & 6.6 & 6.6 & 6.3 & 6.3 & 6.0 & 6.2 & 7.1 & 6.5 & 5.9 & 6.5 & 6.7 & 6.4 & 6.2 & 6.4 \\
\hline \multirow{4}{*}{$\mathrm{X}$} & Long pistil & 7.9 & 8.9 & 7.8 & 8.2 & 8.8 & 8.2 & 7.8 & 8.3 & 7.3 & 8.6 & 8.1 & 8.0 & 8.0 & 8.6 & 7.9 & 8.1 \\
\hline & Medium pistil & 7.3 & 7.7 & 6.7 & 7.2 & 7.2 & 7.6 & 6.4 & 7.1 & 7.2 & 7.9 & 6.8 & 7.3 & 7.2 & 7.7 & 6.7 & 7.2 \\
\hline & Short pistil & 5.6 & 5.7 & 6.1 & 5.8 & 5.6 & 6.0 & 6.0 & 5.9 & 6.0 & 6.0 & 6.0 & 6.0 & 5.8 & 5.9 & 6.1 & 5.9 \\
\hline & $\bar{x}$ & 6.9 & 7.4 & 6.9 & 7.1 & 7.2 & 7.3 & 6.7 & 7.1 & 6.8 & 7.5 & 7.0 & 7.1 & 7.0 & 7.4 & 6.9 & 7.1 \\
\hline
\end{tabular}

LSD0.05

Cultivar (a)

0.40

Pistil length (b)

0.40

Method of pollination (c) ns.

Years (d)

0.40

Interaction (ab)

0.91

(ad) $\quad 0.91$

(bd) $\quad 0.91$ 


\section{DISCUSSION}

The influence of applied operations on flowering of studied eggplant varieties was shown in the experiment performed. Significantly highest mean number of flowers was observed on plants whose flowers were self-pollinated as compared to those whose flowers were hormonized and pollinated by insects. Present paper results correspond to research by Passam et al., (2001) who showed significant reduction of flower number on eggplants due to hormonization. In all pollination ways, there were $42.6-50.9 \%$ flowers with long pistil in whole number of flowers produced on a plant. Much less flowers with medium pistil were observed (20.1-35.1\%) and $22.3-28.9 \%$ of those with short pistil. Górecki and Espinoza-Flores (1996) when investigating the dependence between eggplant's flower structure and fruit setting achieved similar results. Flowers with long pistil were about $63.0 \%$ of total number of flowers produced on a plant and those with medium and short pistil $-15.0 \%$ and $22.0 \%$, respectively. Thus, it can be concluded that application of bumble-bee and flower hormonization in present study did not affect the pistil anatomical structure. Therefore, present paper results does not confirm research by $\mathrm{H}$ an di que and $\mathrm{S}$ arm a (1995) who proved that hormones can modify heterostyly in eggplant's flowers through the impact on flower's anatomical structure and transition of nutrients in pistil's canals. Remarkable role was attributed to auxins (NAA) that influenced on significant reduction of flowers with short pistil, but increased number of formed flowers with long pistil.

Eggplants with flowers with long pistil set the most fruits, then those with medium one and the least-with short pistil. Results of present paper are confirmed by research of Prasada and Prakasha (1968) who showed that eggplants set fruits with long pistil in 70.0-86.7\%, while flowers with short pistil set no fruits. The greatest differences referring to the number of set fruits were observed in flowers with medium pistil (12.5-55.6\%). R y ls ki et al., (1984) achieved similar results recording the highest number of set fruits from flowers with long pistil (90.0\%), while only $5.0 \%$ from flowers with short pistil. Presented results also correspond to research of $\mathrm{P}$ as s a m and Bolmatis (1997) who achieved the highest percentage of set fruits from flowers with long and medium pistil, but flowers with short pistil did not set fruits at all. It should be underlined that fruit setting was significantly reduced in flowers with very long pistil (above $1.2 \mathrm{~cm}$ ). On a base of achieved results, P a s a m and B ol ma tis (1997) found out that although occurrence of flowers with long pistil favors the fruit setting, the short distance between stigmates and anther, not pistil's length in a flower, is more important factor determining the efficient flower pollination.

Different factors affect the dynamics of eggplant's flowers with different-length pistils: genetic basics of a given variety, weather conditions during the cultivation, plant's age and fruiting dynamics. Whether the presence of a bumble-bee stimulates or inhibits the eggplant's flowering remains the unexplained fact. Mean number of flowers produced by three eggplant varieties in object where flowers were pollinated by bumble-bee is unexpectedly low (15.9 flowers per plant) as compared to that observed in plants whose flowers were hormonized ( 24.1 flowers per plant), and in combination with self-pollination (28.9 flowers per plant). 
The following conclusions were formulated:

1. It was found out the influence of pollination ways and flower hormonization on intensity of eggplant's flowering and fruiting. Eggplants formed the most flowers in object with self-pollination and flower hormonization, and those that were pollinated by bumble-bee - much less. Applied operations had not the effects on mean number of set fruits. Pollination of eggplants by bumble-bee affected the increase of the share of set fruit number in flower produced number as compared to the plants whose fruits were set due to flower hormonization and self-pollination.

2. Heterostyly phenomenon occurring in eggplant's flowers affected the plant's fruiting. Regardless the pollination way and flower hormonization, eggplants formed the highest number of flowers with long pistil and much less - with medium and short pistil. The most fruits were set from flowers with long than medium and short pistil.

3. It was shown that the tendency to formation the flowers with particular type of pistil if the variety trait at eggplants. The highest number of flowers with long pistil was observed in varieties 'Solara $\mathrm{F}_{1}$ ' and 'Epic $\mathrm{F}_{1}$ ', and those with medium pistil - on 'Black Beauty' variety.

4. Studied varieties differed referring to total number of flowers and fruits produced. On average, the largest number of flowers was recorded in 'Black Beauty' variety as compared to varieties: 'Solara $\mathrm{F}_{1}$ ' and 'Epic $\mathrm{F}_{1}$ '. Higher number of fruits per plant was achieved from varieties: 'Epic $F_{1}$ ' and 'Solara $F_{1}$ ' than from variety 'Black Beauty'.

5. Heterostyly phenomenon occurring in eggplant's flowers affected the quality of eggplant's fruits. Fruits formed from long pistil flowers were characterized with significantly greater mean weight and size, than those from medium and short pistil ones.

6. Studied varieties differed referring to weight, size and shape of commercial fruits. 'Black Beauty' cv. was distinguished with fruits of much larger weight as compared to 'Epic $F_{1}$ ' and 'Solara $F_{1}$ ' cv. Fruits of 'Solara $F_{1}$ ' and 'Epic $F_{1}$ ' varieties were longer and 'Black Beauty' cv. had greater diameter.

7. Regardless the factors studied, it was proved great influence of weather and thermal conditions present over the cover on eggplant's flowering and fruiting. In combinations with self-pollination and hormonization, plants of three eggplant varieties produced more flowers in 1998, than in 1999 and 2000. In object where flowers were pollinated by bumble-bee, plants cultivated in 1999 were characterized with the highest number of flowers produced as compared to 1998 and 2000. Referring to pollination ways, the highest number of set fruits was achieved in 1999, then in 1998 and 2000. Fruits achieved in 1999, when weather conditions were the most favourable for thermophilic plants, were characterized with the best quality expressing as increased weight, length and diameter as compared to those collected in 1998 and 2000. 


\section{REFERENCES}

A bak K.. Sari M.. Paksoy M., 1995. Efficiency of bumble bees on the vield and quality of eggplant and tomato grown in unheated glasshouses. Acta Hortic., 412: 268-274.

Dobromilska R.. 1997. Plonowanie odmian papryki słodkiej w zaleźności od sposobu zapylania. Mat. VII Ogólnopol. Zjazdu Hod. Roślin Ogrod., 11-13 wrzesień 1997. Szczecin: $399-402$.

Górecki R. S.. Espinosa-Flores A., 1996. Wpływ heterostylii kwiatów oberżyny Solanum melongena L. odm. Rodo na wiązanie owoców oraz liczbę nasion. Mat. Konf. Międzyn. Symp., 15-19 lipiec 1996, Skierniewice: 50-51.

Handique A. K.. Sarma A., 1995. Alteration of heterostyly in Solanum melongena L. through gamma-radiation and hormonal treatment. J. Nuc. Agric. Biol.. 24: 121-126.

Nothmann J., Koller D., 1975. Effects of low-temperature stress on fertility and fruiting of eggplant (Solanum melongena L.) in a subtropical climate. Expl. Agric.. 11: 33-38.

Nothmann J.. Rylski I.. Spigelman M.. 1983. Interactions between floral morphology. position in cluster and 2,4-D treatments in three eggplant cultivars. Sci. Hortic., 20: $35-44$.

Passam H. C.. Bolmat is A.. 1997. The influence of style length on the fruit set. fruit size and seed content of aubergines cultivated under high ambient temperature. Trop. Sci.. 37: 221-227.

Passam H. C.. Baltas C.. Boyiatzoglou A.. Khah E. M.. 2001. Flower morphology and number of aubergine (Solanum melongena L.) in relation to fruit load and auxin application. Sci. Hortic., 89: 309-316.

Prasad D. N.. Prakash R., 1968. Floral biology of brinjal (Solanum melongena L.). I. J. Agric. Sci.. 38: 1053-1061.

Rylski I., Nothmann J., A rcan L., 1984. Differential fertility in short - styled eggplant flowers. Sci. Hortic., 22: 39-46.

Shipp J. L., Whitfield G. H., Papadopoulus A. P., 1994. Effectiveness of the bumblebee. Bombus impatiens Cr. (Hymenoptera:Apidae), as a pollinator of greenhouse sweet pepper. Sci. Hort., 57: 29-39.

\section{Wpływ heterostylii, sposobu zapylania i hormonizacji na kwitnienie i owocowanie oberżyny (Solanum melongena L.)}

\section{Streszczenie}

Doświadczenie przeprowadzono w trzech nieogrzewanych tunelach foliowych w latach 1998-2000. Celem badań była ocena wpływu heterostylii, sposobu zapylania (samozapylenie i zapylanie przez trzmiela) oraz hormonizacji na kwitnienie i owocowanie trzech odmian oberżyny-'Black Beauty', 'Solara $F_{1}$ ' i 'Epic F '. Otrzymane wyniki wykazały. że najwięcej kwiatów wytworzyły rośliny oberżyny w obiek- 
cie z samozapyleniem i hormonizacją kwiatów, zaś znacznie mniej rośliny, których kwiaty zapylały trzmiele. Niezależnie od sposobu zapylania i hormonizacji kwiatów rośliny oberżyny wytworzyły najwięcej kwiatów ze słupkiem wysokim, zaś zdecydowanie mniej ze słupkiem pośrednim i niskim. Wykazano, że tendencja do tworzenia kwiatów o poszczególnych rodzajach słupka jest cechą odmianową oberżyny. Na roślinach odmian: 'Solara $\mathrm{F}_{1}$ ' i 'Epic $\mathrm{F}_{1}$ ' zaobserwowano najwięcej kwiatów ze słupkiem wysokim, zaś na roślinach odmiany 'Black Beauty' ze słupkiem pośrednim.

Zjawisko heterostylii występujące w kwiatach oberżyny miało wpływ na owocowanie roślin. Więcej owoców powstało z kwiatów ze słupkiem wysokim, aniżeli z kwiatów ze słupkiem pośrednim i niskim. Owoce powstałe z kwiatów o słupku wysokim odznaczały się istotnie większą masą i wielkością, niż uzyskane z kwiatów o słupku pośrednim i niskim.

W trzech latach badań nie stwierdzono istotnego wpływu zastosowanych zabiegów na jakość owoców oberżyny. Owoce uzyskane we wszystkich sposobach zapylania charakteryzowały się podobną średnią masą i średnicą. Z roślin, których kwiaty zapylane były przez trzmiela uzyskano owoce o istotnie większej średniej długości, aniżeli z roślin, których kwiaty zapylały się same. 